bo um 4,7 Punkte. Die Patienten konnten dabei besser ein- und durchschlafen und mussten nachts seltener auf die Toilette, auch die morgendliche Akinese und die Tagesschläfrigkeit waren weniger stark ausgeprägt als in der Placebogruppe.

\section{Spezielle Galenik}

Ermöglicht wird die kontinuierliche Wirkstofffreisetzung durch das dreischichtige Geomatrix ${ }^{\circledR}$-System. Zwei semipermeable Barriereschichten umhüllen dabei einen Matrixkern, der im Darm aufquillt und Ropinirol kontrolliert abgibt. Aufgrund dieser speziellen Galenik gebe es deutliche Unterschiede $z$ wischen ReQuip-Modutab ${ }^{\oplus}$ und retardierten Ropinirol-Generika, so Reichmann. Der Neurologe warnte davor, gut ein- gestellte Parkinson-Patienten auf Generika umzustellen und erinnerte an die Leitlinie der Deutschen Pharmazeutischen Gesellschaft zur "Guten Substitutionspraxis". Nach dieser soll bei Patienten, die eine kontinuierliche Therapie und eine besonders gute Einstellung benötigen, auf einen häufigen Präparatewechsel verzichtet werden. Da das Ropinirol-Originalpräparat seit über einem Jahr zum Festbetrag erhältlich ist, gebe es für eine Umstellung auch wenig Gründe.

Thomas Müller

Pressegespräch „Individualisierte ParkinsonTherapie: Chancen erkennen und Potenziale nutzen", DGN-Kongress, Hamburg, 26.9.12 Veranstalter: GlaxoSmithKline

\title{
Bei MS frühzeitig an das langfristige Outcome denken
}

— Bei der schubförmigen Multiplen Sklerose (RRMS) sollte man mit einer Eskalationstherapie nicht zu lange warten, wenn der Patient trotz Basistherapie weiter Schubaktivität zeigt. Denn auch in diesem Krankheitsstadium gebe es ein „Window of Opportunity", in dem die Chancen am größten seien, die Krankheitsaktivität anhaltend und deutlich zu reduzieren, mahnte Professor HansPeter Hartung, Neurologische Universitätsklinik Düsseldorf.

Dass auch in der Eskalationstherapie ein solches Zeitfenster existiert, innerhalb dessen Patienten mit hoher Krankheitsaktivität von einer rechtzeitigen Umstellung am meisten profitieren, zeigen laut Hartung die inzwischen bis zu einer Therapiedauer von sieben Jahren vorliegenden Extensionsstudien für Fingolimod (Gilenya ${ }^{\oplus}$ ). Es komme darauf an, die Entscheidung rasch zu fällen, wenn der Patient die Indikation für eine Eskalationstherapie erfülle. Die Basistherapie weiter zu führen und auf einen Therapieerfolg zu hoffen, sei die falsche Strategie, so der MS-Experte.

Die Verläufe in den großen Phase-III-Studien (FTY720 Research Evaluating Effects of Daily Oral Therapy in Multiple Sclerosis; $\mathrm{n}=1272$ und FREEDOMS II; $\mathrm{n}=1083$ ) nach zwei und vier Jahren zeigen, so Hartung, dass der frühzeitige Einsatz von Fingolimod Vorteile bietet. Denn die frühzeitig mit Fingolimod be- handelten Patienten hätten im Vergleich zu den erst später von Placebo auf Fingolimod umgestellten Patienten eine anhaltend geringere Schubaktivität und weniger Krankheitszeichen im MRT aufgewiesen. Die Extensionsstudie derTRANSFORMS-Basisstudie (Trial Assessing Injectable Interferon vs. FTY720 Oral in RRMS) habe nach 4,5 Jahren zudem gezeigt, dass die weiterhin mit Interferon-beta 1a i.m. behandelten Patienten schlechter abschneiden als die mit Fingolimod behandelten Patienten.

In einer Analyse der mit Fingolimod durchgeführten Phase-II/III-Basisstudien und Extensionsstudien wurden die Sicherheitsdaten von 3.553 Teilnehmern ausgewertet, was 9.070 Patientenjahren (alle Dosierungen) entspricht. Die Daten zeigen keinen Hinweis, dass sich das Sicherheitsprofil mit wachsender Behandlungsdauer verschlechtert, sagte Hartung. Dies gelte auch für das Auftreten von Infektionen, Blutdruckerhöhungen, kardialen Effekten und Malignomen.

Dr. Alexander Kretzschmar

Satellitensymposium "Growing global experience with once-daily oral fingolimod in $\mathrm{MS}^{\prime \prime}$ im Rahmen der Jahrestagung der European Committee for Treatment and Research in Multiple Sclerosis (ECTRIMS) 2012,

Lyon, 10.10.2012

Veranstalter: Novartis Pharma GmbH

\section{Alzheimer-Demenz}

Seit Mitte Dezember 2012 ist Memantine Merz ${ }^{\circledast}$ auf dem Markt verfügbar. Das Axura ${ }^{\circledR}$-identische Generikum zur Behandlung der moderaten bis schweren Alzheimer-Demenz wird in den gleichen Dosierungen und Packungsgrößen angeboten wie das Original Axura ${ }^{\oplus}$, auch die Galenik ist identisch, Farbe und Form der Tabletten ändern sich nicht.

Nach Informationen von Merz

\section{ADHS-Förderpreis verliehen}

Den diesjährigen ADHS-Förderpreis teilen sich die von Professor Hans Biegert geleitete HEBO-Privatschule Bonn und das Private Gymnasium Esslingen unter der Leitung von Thomas Dahm. Einen Sonderpreis erhielt der Emdener Kinderarzt Götz Gnielka für sein Projekt ,aus der Kita in die Schule - $A D(H) S$ früh erkennen - sicher begleiten". Der vom Unternehmen Shire verliehene Preis ist mit $10.000 €$ dotiert.

Nach Informationen von Shire

\section{App für Patienten mit Depression}

Das Unternehmen Lundbeck stellt die App "Back to Job" für Patienten mit Depression ab sofort zur Verfügung. Die Anwendung für das iPhone kann kostenlos im AppStore heruntergeladen werden. Die App unterstützt Patienten bei der regelmäBigen Dokumentation ihrer Gefühlslage, gibt Tipps für den Alltag und bietet die Möglichkeit, die Dokumentation mit dem behandelnden Arzt abzustimmen. Mittels einer Kalenderfunktion können die Patienten einzelne Parameter der Krankheit nach dem Schulnotensystem bewerten. Beurteilt werden die Stimmung zur aktuellen Tageszeit, das Maß an Freude und Interesse, sowie Energie und Antrieb, das körperliche Befinden, die Schlafqualität und das aktuelle Befinden. Die Daten werden als Grafik für verschiedene Zeiträume ausgegeben und können per E-Mail an den Arzt gesandt werden. Dieser kann so etwaige Änderungen im Affekt erkennen und die Behandlung des Patienten gegebenenfalls individuell anpassen. Berufstätigen Patienten mit Depression, die ihr Smartphone immer bei sich haben, kann die App zusätzlich helfen, Ihren Alltag zu strukturieren. Die App soll in Kürze auch für Android-Systeme verfügbar werden.

Nach Informationen von Lundbeck 